\title{
RESOLVENT ESTIMATES \\ FOR ELLIPTIC FINITE ELEMENT OPERATORS IN ONE DIMENSION
}

\author{
M. CROUZEIX, S. LARSSON, AND V. THOMÉE
}

\begin{abstract}
We prove the analyticity (uniform in $h$ ) of the semigroups generated on $L_{p}(0,1), 1 \leq p \leq \infty$, by finite element analogues $A_{h}$ of a onedimensional second-order elliptic operator $A$ under Dirichlet boundary conditions. This is accomplished by showing the appropriate estimates for the resolvents by means of energy arguments. The results are applied to prove stability and optimal-order error bounds for numerical solutions of the associated parabolic problem for both smooth and nonsmooth data.
\end{abstract}

\section{INTRODUCTION}

Let $A$ be an elliptic differential operator in one dimension defined by

$$
A u=-\left(a u^{\prime}\right)^{\prime}+b u^{\prime}+c u, \quad x \in \Omega \equiv(0,1), \text { with } u(0)=u(1)=0,
$$

where $u^{\prime}=d u / d x$ and the coefficients $a, b, c$ are smooth and real-valued with $a$ positive on $\bar{\Omega}$. Defining the corresponding sesquilinear form

$$
A(u, v)=\left(a u^{\prime}, v^{\prime}\right)+\left(b u^{\prime}, v\right)+(c u, v), \quad \text { where }(u, v)=\int_{0}^{1} u \bar{v} d x
$$

we assume that there is $a_{0}>0$ such that

$$
\operatorname{Re} A(v, v) \geq a_{0}\left\|v^{\prime}\right\|^{2}, \quad v \in H_{0}^{1}(\Omega),
$$

where $\|u\|^{2}=(u, u)$. If this is not satisfied originally, it may be accomplished by adding a positive multiple of $u$ to $A u$.

Let $0=x_{0}<x_{1}<\cdots<x_{N}<x_{N+1}=1$ define a partition of the interval $\Omega$ into subintervals $\Omega_{i}=\left(x_{i}, x_{i+1}\right)$ of lengths $h_{i}=x_{i+1}-x_{i}$ and with $h=\max h_{i}$. We shall think of this partition as a member of a family of such partitions, which is assumed to be quasi-uniform in the sense that for some $c>0$ we have $h_{i} \geq c h, i=0,1, \ldots, N$. For a fixed integer $r \geq 2$, we define

$$
S_{h}=\left\{v \in H_{0}^{1}(\Omega):\left.v\right|_{\Omega_{i}} \in \Pi_{r-1}, i=0,1, \ldots, N\right\},
$$

Received by the editor March 31, 1993.

1991 Mathematics Subject Classification. Prmary 65M60, 65M12.

Key words and phrases. Resolvent, sectorial, elliptic, analytic semigroup, Banach space, $L_{p}$, maximum norm, finite element method, rational approximation, stability, error estimate.

The second and third authors were partly supported by the Swedish Research Council for Engineering Sciences (TFR). 
where $\Pi_{r-1}$ denotes the space of all polynomials (with complex coefficients) of degree $\leq r-1$. We then define a discrete analogue $A_{h}: S_{h} \rightarrow S_{h}$ of the operator $A$ by the relation

$$
\left(A_{h} \psi, \chi\right)=A(\psi, \chi), \quad \forall \psi, \chi \in S_{h} .
$$

Let $R(\lambda,-A)=(\lambda I+A)^{-1}$, where $\lambda$ is a complex parameter, denote the resolvent of $-A$. More precisely, we define $R(\lambda,-A) f$ for $f \in H^{-1}(\Omega)$, the dual space of $H_{0}^{1}(\Omega)$, as the solution $u=u(\cdot, \lambda) \in H_{0}^{1}(\Omega)$ of the variational equation

$$
A(u, \chi)+\lambda(u, \chi)=\langle f, \bar{\chi}\rangle, \quad \forall \chi \in H_{0}^{1}(\Omega),
$$

where $\langle\cdot, \cdot\rangle$ denotes the duality pairing between $H^{-1}(\Omega)$ and $H_{0}^{1}(\Omega)$. Similarly, we note that $R\left(\lambda,-A_{h}\right) P_{h} f$ is the solution $u_{h}=u_{h}(\cdot, \lambda) \in S_{h}$ of

$$
A\left(u_{h}, \chi\right)+\lambda\left(u_{h}, \chi\right)=\langle f, \bar{\chi}\rangle, \quad \forall \chi \in S_{h},
$$

if we define the projection $P_{h}: H^{-1}(\Omega) \rightarrow S_{h}$ by

$$
\left(P_{h} f, \chi\right)=\langle f, \bar{\chi}\rangle, \quad \forall \chi \in S_{h} .
$$

Note that the restriction of $P_{h}$ to $L_{2}(\Omega)$ is the standard orthogonal projection.

The main purpose of this work is to prove the following estimates of the resolvents of $-A$ and $-A_{h}$. We use the notation $\|\cdot\|_{p}$ for the standard norms in $L_{p}(\Omega), 1 \leq p \leq \infty$.

Theorem 1.1. There are $\varphi \in\left(\frac{1}{2} \pi, \pi\right)$ and $C \geq 1$ such that the linear operators $R(\lambda,-A)$ and $R\left(\lambda,-A_{h}\right) P_{h}$ are bounded from $H^{-1}(\Omega)$ into $H_{0}^{1}(\Omega)$, uniformly with respect to $\lambda$ in the sector $\Sigma_{\varphi}=\{\lambda \in \mathbf{C}:|\arg \lambda| \leq \varphi\}$, and, for $1 \leq p \leq \infty$, we have

$$
\|R(\lambda,-A) f\|_{p}+\left\|R\left(\lambda,-A_{h}\right) P_{h} f\right\|_{p} \leq \frac{C}{1+|\lambda|}\|f\|_{p}, \quad f \in L_{p}(\Omega), \lambda \in \Sigma_{\varphi} .
$$

This theorem is proved in $\S 2$. The proof is based on estimates of the Green's functions of (1.2) and (1.3), which we obtain by an energy argument. Since the relevant Green's functions do not belong to $H_{0}^{1}(\Omega)$ in the multidimensional case, it is not clear whether this technique can be generalized; onedimensionality is also used in various technical details below.

In the final $\S 3$ we discuss some applications of these resolvent estimates. The first application concerns stability and error estimates for spatially semidiscrete finite element approximations of the parabolic initial-boundary value problem

$$
\begin{array}{ll}
u_{t}+A u=f(x, t), & x \in \Omega, t>0, \\
u(0, t)=u(1, t)=0, & t>0, \\
u(x, 0)=u_{0}(x), & x \in \Omega,
\end{array}
$$

where $u=u(x, t), u_{t}=\partial u / \partial t$, and where $f$ and $u_{0}$ are given. The semidiscrete finite element approximation $u_{h}(t) \in S_{h}$ is defined by the equation

$$
u_{h, t}+A_{h} u_{h}=P_{h} f(\cdot, t), \quad t>0 ; \quad u_{h}(0)=u_{0 h},
$$

where $u_{0 h} \in S_{h}$ is an approximation of $u_{0}$. 
We now think of $A$ as an unbounded operator on $L_{p}(\Omega)$ with domain $\mathscr{D}(A)=\dot{W}_{p}^{2}(\Omega)=\left\{u \in W_{p}^{2}(\Omega): u(0)=u(1)=0\right\}$. Elementary arguments show that

$$
\|u\|_{m, p} \leq C_{m}\|A u\|_{m-2, p}, \quad u \in W_{p}^{m}(\Omega) \cap \dot{W}_{p}^{2}(\Omega), \quad m \geq 2,
$$

where $\|u\|_{m, p}=\left(\sum_{j=0}^{m}\left\|u^{(j)}\right\|_{p}^{p}\right)^{1 / p}$ denotes the standard norm in $W_{p}^{m}(\Omega)$ (with the usual modification for $p=\infty$ ), and hence that $A$ is a closed operator on $L_{p}(\Omega)$. Together with the resolvent estimates in Theorem 1.1, this shows that $A$ and $A_{h}$ generate analytic semigroups $E(t)=e^{-t A}$ and $E_{h}(t) P_{h}=e^{-t A_{h}} P_{h}$ in $L_{p}(\Omega)$; see, for example, [12, Theorem 2.5.2]. In the usual way we obtain the representation

$$
E(t)=e^{-t A}=\frac{1}{2 \pi i} \int_{\Gamma} e^{t \lambda} R(\lambda,-A) d \lambda,
$$

where the contour $\Gamma$ is the boundary of $\Sigma_{\varphi}$ oriented so that $\operatorname{Im} \lambda$ increases along $\Gamma$, and similarly

$$
E_{h}(t)=e^{-t A_{h}}=\frac{1}{2 \pi i} \int_{\Gamma} e^{t \lambda} R\left(\lambda,-A_{h}\right) d \lambda
$$

Using (1.8), (1.9) and the resolvent estimates, we obtain the bounds

$$
\|E(t) v\|_{p}+t\|A E(t) v\|_{p} \leq C\|v\|_{p}, \quad t>0,
$$

and

$$
\left\|E_{h}(t) P_{h} v\right\|_{p}+t\left\|A_{h} E_{h}(t) P_{h} v\right\|_{p} \leq C\|v\|_{p}, \quad t>0,
$$

by a standard argument. In a similar way we see that $E(t)$ and $E_{h}(t) P_{h}$ are bounded from $H^{-1}(\Omega)$ into $H_{0}^{1}(\Omega)$ for $t>0$; in the latter case the bound is independent of $h$. From semigroup theory it also follows that $u(t)=E(t) u_{0}$ and $u_{h}(t)=E_{h}(t) u_{0 h}$ are solutions of the homogeneous problems (1.5) and (1.6) with $f(x, t) \equiv 0$. Solutions of the nonhomogeneous problems can then be obtained by Duhamel's principle: for (1.5) we have

$$
u(t)=E(t) u_{0}+\int_{0}^{t} E(t-s) f(\cdot, s) d s, \quad t \geq 0,
$$

under suitable regularity assumptions on $f$. An analogous formula holds for (1.6).

In this connection we note that when $p=\infty$ we have $\overline{\mathscr{D}(A)}=C_{0}(\bar{\Omega})=$ $\{u \in C(\bar{\Omega}): u(0)=u(1)=0\}$, so that $A$ is not densely defined in $L_{\infty}(\Omega)$, and hence the standard theory of analytic semigroups does not apply when $p=\infty$. However, most of the semigroup theory can be developed without the assumption that $\mathscr{D}(A)$ is dense; see [13] and [6]. Note also that $A$ is densely defined in $C_{0}(\bar{\Omega})$ with the maximum norm; in that space the classical semigroup theory is thus applicable. But the existence theorems for (1.12) then require that $f(t) \in C_{0}(\bar{\Omega})$, which is not satisfactory, since it places an unnecessary boundary condition on $f(t)$. We therefore prefer to work in $L_{\infty}(\Omega)$ rather than in $C_{0}(\bar{\Omega})$.

Stability estimates of the form (1.11), with $p=\infty$, but with logarithmic factors in the right-hand sides, were obtained in [15] and [14]. Apart from 
removing the logarithm, our present proof of stability is simpler than those of $[15,14]$.

Using the stability estimate for $E_{h}(t)$ in (1.11) we prove in Theorem 3.2 that for $u_{0 h}$ suitably chosen we have the error estimate

$$
\left\|u_{h}(t)-u(t)\right\|_{p} \leq C h^{r}\left(\left\|u_{0}\right\|_{r, p}+\int_{0}^{t}\left\|u_{t}(s)\right\|_{r, p} d s\right), \quad 1 \leq p \leq \infty .
$$

Smooth solutions are thus approximated to optimal order.

In some applications it is important to allow nonsmooth initial data, such as discontinuous functions or even $\delta$-functions. Let $\mathscr{M}(\Omega)$ denote the set of finite measures $\mu$ with $\|\mu\|_{\mathscr{M}}=|\mu|(\Omega)=\int_{\Omega} d|\mu|$. With each $\mu \in \mathscr{M}(\Omega)$ we associate a distribution (also denoted $\mu$ ) in the usual way: $\langle\mu, \chi\rangle=\int_{\Omega} \chi(x) d \mu(x)$ for $\chi \in C(\Omega)$. Recall that $P_{h}: \mathscr{M}(\Omega) \rightarrow S_{h}$ is defined in (1.4). For solutions of the homogeneous equations, i.e., (1.5) and (1.6) with $f(x, t) \equiv 0$, and with $u_{0 h}=P_{h} u_{0}$, we show in Theorem 3.5 that, for $t>0$,

$$
\begin{array}{rlrl}
\left\|u_{h}(t)-u(t)\right\|_{p} & \leq C h^{r} t^{-r / 2}\left\|u_{0}\right\|_{p}, & & u_{0} \in L_{p}(\Omega), \quad 1 \leq p \leq \infty, \\
\left\|u_{h}(t)-u(t)\right\|_{\infty} \leq C h^{r} t^{-(r+1) / 2}\left\|u_{0}\right\|_{\mathscr{M}}, & & u_{0} \in \mathscr{M}(\Omega) .
\end{array}
$$

In addition to (1.10) and (1.11) the proof uses a stability property of $E(t)$ and $E_{h}(t)$ considered as operators from $\mathscr{M}(\Omega)$ to $L_{\infty}(\Omega)$, namely

$$
\|E(t) v\|_{\infty}+\left\|E_{h}(t) P_{h} v\right\|_{\infty} \leq C t^{-1 / 2}\|v\|_{\mathscr{M}}, \quad t>0,
$$

resulting from the corresponding resolvent estimates, which are also derived in $\S 2$.

We remark that our proofs of these error bounds differ slightly from those of [14], which introduce additional logarithmic factors.

Another application of the resolvent estimates in Theorem 1.1 concerns the stability and error analysis of fully discrete schemes based on rational approximations of the analytic semigroup $E_{h}(t)$. Let $r(\lambda)$ be a rational approximation of the exponential function $\exp (\lambda)$, which is accurate of order $q \geq 1$, i.e.,

$$
r(\lambda)=e^{\lambda}+O\left(\lambda^{q+1}\right), \quad \lambda \rightarrow 0,
$$

and $A$-acceptable, i.e.,

$$
|r(\lambda)| \leq 1, \quad \operatorname{Re} \lambda \leq 0 .
$$

Then $E_{k h}^{n} u_{0 h}=r\left(-k A_{h}\right)^{n} u_{0 h}$ is an approximation of $E_{h}\left(t_{n}\right) u_{0 h}$, where $k$ is the timestep and $t_{n}=n k$ for $n=1,2, \ldots$. Using the resolvent estimate and contour integral representations, one may then prove stability for the discrete evolution operator $E_{k h}^{n}$,

$$
\left\|E_{k h}^{n} u_{0 h}\right\|_{p} \leq C\left\|u_{0 h}\right\|_{p}, \quad n \geq 0, \quad 1 \leq p \leq \infty,
$$

see $[3,10,11]$. Stability may be combined with a local truncation error analysis in a similar way as in (1.13) to yield an error bound for certain completely discrete schemes. We demonstrate this in Theorem 3.3 for the backward Euler method:

$$
\begin{aligned}
& U_{n} \in S_{h}, U_{0}=u_{0 h}, \\
& \bar{\partial}_{t} U_{n}+A_{h} U_{n}=P_{h} f\left(t_{n}\right), \quad \bar{\partial}_{t} U_{n}=\left(U_{n}-U_{n-1}\right) / k, \quad n \geq 1,
\end{aligned}
$$


and the Crank-Nicolson method:

$$
\begin{aligned}
& U_{n} \in S_{h}, U_{0}=u_{0 h}, \\
& \bar{\partial}_{t} U_{n}+A_{h}\left(U_{n}+U_{n-1}\right) / 2=P_{h} f\left(t_{n-1 / 2}\right), \quad n \geq 1 .
\end{aligned}
$$

For general time discretization schemes it is necessary to introduce more complicated approximations of the source term $f$; see [2]. We refrain from addressing this difficulty here and restrict further discussion to the homogeneous problem $(f \equiv 0)$. In this case we have $U_{n}=E_{k h}^{n} u_{0 h}, u_{h}(t)=E_{h}(t) u_{0 h}$, and the resolvent estimates and contour integral representations yield the error bounds

$$
\begin{aligned}
& \left\|U_{n}-u_{h}\left(t_{n}\right)\right\|_{p} \leq C k^{j}\left\|A_{h}^{j} u_{0 h}\right\|_{p}, \\
& \quad t_{n}=n k \geq 0,1 \leq p \leq \infty, 1 \leq j \leq q,
\end{aligned}
$$

and, if $r$ is strongly $A$-acceptable, that is, if in addition $|r(\infty)|<1$, then

$$
\left\|U_{n}-u_{h}\left(t_{n}\right)\right\|_{p} \leq C k^{q} t_{n}^{-q}\left\|u_{0 h}\right\|_{p}, \quad t_{n}=n k>0,1 \leq p \leq \infty,
$$

see for example [8]. Using the above bounds for $u_{h}(t)-u(t)$ and some additional arguments, one may then obtain bounds for the total error $U_{n}-u\left(t_{n}\right)$. For example, when $u_{0 h}=P_{h} u_{0}$, the error bounds (1.14) and (1.21) immediately give

$$
\left\|U_{n}-u\left(t_{n}\right)\right\|_{p} \leq C\left(h^{r} t_{n}^{-r / 2}+k^{q} t_{n}^{-q}\right)\left\|u_{0}\right\|_{p}, \quad t_{n}=n k>0, \quad 1 \leq p \leq \infty,
$$

because $P_{h}$ is stable in $L_{p}(\Omega)$ by a result of [4]. We may also obtain error bounds which hold uniformly as $t \rightarrow 0$, although an argument based directly on (1.20) is not satisfactory, since this applies a discrete norm to the discrete initial value. We show in Theorem 3.6 by a somewhat more involved argument that

$$
\begin{aligned}
& \left\|U_{n}-u\left(t_{n}\right)\right\|_{p} \leq C\left(h^{r}\left\|u_{0}\right\|_{r, p}+k^{q}\left\|u_{0}\right\|_{2 q, p}\right), \\
& \quad t_{n}=n k \geq 0, \quad 1 \leq p \leq \infty,
\end{aligned}
$$

if $f \equiv 0, u_{0}$ is sufficiently smooth and satisfies the appropriate compatibility conditions at $x=0,1$, and $u_{0 h}$ is suitably chosen.

\section{Resolvent estimates}

The main object of this section is to prove the bounds in Theorem 1.1 for the resolvents $R(\lambda,-A)$ and $R\left(\lambda,-A_{h}\right)$. The resolvent operators will be studied for $\lambda$ in a sector

$$
\Sigma_{\kappa, \theta}=\{\lambda \in \mathbf{C}:|\arg (\lambda-\kappa)| \leq \theta\}, \quad \text { with } \kappa \geq 0, \theta \in\left(\frac{1}{2} \pi, \pi\right) .
$$

For $\lambda \in \Sigma_{\kappa, \theta}$ it is convenient to write

(2.1) $\lambda-\kappa=(\xi+i \eta)^{2}=\xi^{2}-\eta^{2}+2 i \xi \eta, \quad$ with $\xi+i \eta \in \Sigma_{0, \theta / 2}, \xi, \eta \in \mathbf{R}$.

For future reference we note that for $\lambda \in \Sigma_{\kappa, \theta}$, we have

$$
|\eta| \leq \delta \xi, \quad \text { where } \delta=\tan \left(\frac{1}{2} \theta\right)>1,
$$

and

$$
\xi^{2} \leq|\lambda-\kappa|=\xi^{2}+\eta^{2} \leq\left(1+\delta^{2}\right) \xi^{2} .
$$

The following estimate will be a basic tool. 
Lemma 2.1. For any $\theta \in\left(\frac{1}{2} \pi, \pi\right)$ there are $\kappa \geq 0$ and $C \geq 1$ such that

$$
\left\|v^{\prime}\right\|^{2}+\xi^{2}\|v\|^{2} \leq C\left|A(v, v)+\lambda\|v\|^{2}\right|, \quad v \in H_{0}^{1}(\Omega), \lambda \in \Sigma_{\kappa, \theta},
$$

where $\xi$ is related to $\lambda$ as in (2.1). If $A$ is selfadjoint, i.e., $b(x) \equiv 0$, then we may choose $\kappa=0$.

Proof. Setting

$$
F=A(v, v)+\lambda\|v\|^{2}
$$

we obtain, by taking the real part of (2.5) and using the transformation of variables in (2.1),

$$
\operatorname{Re} A(v, v)+\left(\kappa+\xi^{2}-\eta^{2}\right)\|v\|^{2}=\operatorname{Re} F,
$$

and together with (1.1),

$$
a_{0}\left\|v^{\prime}\right\|^{2}+\left(\kappa+\xi^{2}-\eta^{2}\right)\|v\|^{2} \leq|F|
$$

By taking the imaginary part of (2.5) we obtain

$$
\operatorname{Im} A(v, v)+2 \xi \eta\|v\|^{2}=\operatorname{Im} F,
$$

and, since $\operatorname{Im} A(v, v)=\operatorname{Im}\left(b v^{\prime}, v\right)$,

$$
2 \xi|\eta|\|v\|^{2} \leq|F|+\|b\|_{\infty}\left\|v^{\prime}\right\|\|v\| .
$$

Multiplying by $\frac{1}{2} \delta=\frac{1}{2} \tan \left(\frac{1}{2} \theta\right)$ we have, in view of (2.2),

$$
\eta^{2}\|v\|^{2} \leq \delta \xi|\eta|\|v\|^{2} \leq \frac{1}{2} \delta|F|+\frac{1}{2} \delta\|b\|_{\infty}\left\|v^{\prime}\right\|\|v\| .
$$

Adding this to (2.6), we obtain

$$
a_{0}\left\|v^{\prime}\right\|^{2}+\left(\kappa+\xi^{2}\right)\|v\|^{2} \leq\left(1+\frac{1}{2} \delta\right)|F|+\frac{1}{2} a_{0}\left\|v^{\prime}\right\|^{2}+\frac{\delta^{2}\|b\|_{\infty}^{2}}{8 a_{0}}\|v\|^{2},
$$

and (2.4) follows by taking $\kappa=\delta^{2}\|b\|_{\infty}^{2} /\left(8 a_{0}\right)$.

If $f \in L_{2}(\Omega)$, then taking $\chi=u$ in (1.2) and applying the lemma shows

$$
\left\|u^{\prime}\right\|^{2}+\xi^{2}\|u\|^{2} \leq C|(f, u)| \leq C\|f\|\|u\|, \quad \lambda \in \Sigma_{\kappa, \theta} .
$$

In particular, using (2.3), we have

$$
\|R(\lambda,-A) f\| \leq \frac{C}{\xi^{2}}\|f\| \leq \frac{C}{|\lambda-\kappa|}\|f\|, \quad \lambda \in \Sigma_{\kappa, \theta},
$$

which shows that $-A$ is a sectorial operator on $L_{2}(\Omega)$. The same argument applies to equation (1.3), so that $-A_{h}$ is also sectorial, uniformly in $h$, with respect to the topology of $L_{2}(\Omega)$.

We next prove that $-A$ is sectorial on $L_{p}(\Omega)$ for $1 \leq p \leq \infty$. This result is well known, but we give an elementary proof based on the energy estimate of Lemma 2.1 , which we shall then modify in Theorem 2.3 to show that $-A_{h}$ is sectorial in the topology of $L_{p}(\Omega)$ uniformly in $h$. 
Theorem 2.2. For any $\theta \in\left(\frac{1}{2} \pi, \pi\right)$ there are $\kappa \geq 0$ and $C \geq 1$ such that

$$
\|R(\lambda,-A) f\|_{p} \leq \frac{C}{|\lambda-\kappa|}\|f\|_{p}, \quad f \in L_{p}(\Omega), \lambda \in \Sigma_{\kappa, \theta}, \quad 1 \leq p \leq \infty .
$$

Proof. Let $\theta \in\left(\frac{1}{2} \pi, \pi\right)$ be arbitrary and let $\kappa$ be as in Lemma 2.1. Lemma 2.1 implies that the sesquilinear form $B_{\lambda}(u, v)=A(u, v)+\lambda(u, v)$ is coercive on $H_{0}^{1}(\Omega)$, that is, $\left|B_{\lambda}(u, u)\right| \geq C^{-1}\left\|u^{\prime}\right\|^{2}$ for all $u \in H_{0}^{1}(\Omega)$ and $\lambda \in \Sigma_{\kappa, \theta}$. Hence, by the Lax-Milgram lemma we conclude that (1.2) has a unique solution and that

$$
\|R(\lambda,-A) f\|_{H_{0}^{1}} \leq C\|f\|_{H^{-1}}, \quad f \in H^{-1}(\Omega), \lambda \in \Sigma_{\kappa, \theta} .
$$

We shall prove the resolvent estimate for $p=\infty$. Since $L_{1}(\Omega)^{*}=L_{\infty}(\Omega)$ and $R(\lambda,-A)^{*}=R\left(\bar{\lambda},-A^{*}\right)$, where the adjoint operator $A^{*}$ is of the same form as $A$, the estimate then follows for $p=1$ by duality and for $1<p<\infty$ by interpolation.

To complete the proof, it thus remains to show

$$
\|u(\cdot, \lambda)\|_{\infty} \leq \frac{C}{|\lambda-\kappa|}\|f\|_{\infty}, \quad \lambda \in \Sigma_{\kappa, \theta},
$$

where $u(\cdot, \lambda) \in H_{0}^{1}(\Omega)$ is the solution of equation (1.2) with $f \in L_{\infty}(\Omega)$. Let $g=g(\cdot, y, \lambda) \in H_{0}^{1}(\Omega)$ be the Green's function of the adjoint operator $A^{*}+\bar{\lambda} I$ with singularity at the point $y \in \Omega$, i.e.,

$$
A(\chi, g)+\lambda(\chi, g)=\chi(y), \quad \forall \chi \in H_{0}^{1}(\Omega) .
$$

Since by (1.2) and (2.8) we have $u(y, \lambda)=\langle f, \overline{g(\cdot, y, \lambda)}\rangle=(f, g(\cdot, y, \lambda))$, the estimate (2.7) follows if we show

$$
\|g(\cdot, y, \lambda)\|_{1} \leq \frac{C}{|\lambda-\kappa|}, \quad y \in \Omega, \lambda \in \Sigma_{\kappa, \theta} .
$$

Our strategy for proving (2.9) is to introduce the function

$$
v(x)=v(x, y, \lambda)=g(x, y, \lambda) e^{\gamma \xi|x-y|},
$$

where $\xi$ is related to $\lambda$ as in (2.1) and $\gamma$ is a positive number to be chosen below. We shall prove

$$
\|v(\cdot, y, \lambda)\|_{\infty} \leq C \xi^{-1}, \quad y \in \Omega, \lambda \in \Sigma_{\kappa, \theta},
$$

which implies

$$
|g(x, y, \lambda)| \leq C \xi^{-1} e^{-\gamma \xi|x-y|}, \quad x, y \in \Omega, \lambda \in \Sigma_{\kappa, \theta},
$$

and (2.9) follows in view of (2.3). The Ansatz (2.10) is motivated by the observation that, in the special case when $A u=-u^{\prime \prime}$, we have

$$
0 \leq g\left(x, y, \xi^{2}\right) \leq \frac{1}{2} \xi^{-1} e^{-\xi|x-y|}, \quad \xi>0,
$$

by the maximum principle, the bound being a fundamental solution. We also remark that $g(x, y, \lambda)$ may be calculated explicitly in this case to give a direct proof of (2.9); see [5, $\S 8.1]$.

To complete the proof, it thus remains to prove (2.11). From (2.10) we have $g(x)=v(x) e^{-\gamma \xi|x-y|}$, so that

$$
g^{\prime}(x)=v^{\prime}(x) e^{-\gamma \xi|x-y|}-\gamma \xi v(x) e^{-\gamma \xi|x-y|} s(x-y),
$$


where $s(x)= \pm 1$ according to the sign of $x$. Choosing $\chi(x)=v(x) e^{\gamma \xi|x-y|}$ for the test function in (2.8), we obtain by straightforward calculations that

$$
\begin{aligned}
A(v, v)+\lambda\|v\|^{2}= & v(y)+2 i \gamma \xi \operatorname{Im}\left(a s(\cdot-y) v^{\prime}, v\right) \\
& +\gamma^{2} \xi^{2}(a v, v)-\gamma \xi(b s(\cdot-y) v, v) .
\end{aligned}
$$

In order to apply Lemma 2.1, we need to bound the right-hand side. First we use the inequality

$$
\|v\|_{\infty} \leq \sqrt{\left\|v^{\prime}\right\|\|v\|}, \quad v \in H_{0}^{1}(\Omega)
$$

to get

$$
|v(y)| \leq \sqrt{\left\|v^{\prime}\right\|\|v\|} \leq \frac{1}{2} \gamma^{-1} \xi^{-1}+\frac{1}{4}\left\|v^{\prime}\right\|^{2}+\frac{1}{4} \gamma^{2} \xi^{2}\|v\|^{2} .
$$

Next we have

$$
2 \gamma \xi\left|\operatorname{Im}\left(a s(\cdot-y) v^{\prime}, v\right)\right| \leq 2 \gamma \xi\|a\|_{\infty}\left\|v^{\prime}\right\|\|v\| \leq \frac{1}{4}\left\|v^{\prime}\right\|^{2}+C \gamma^{2} \xi^{2}\|v\|^{2} .
$$

Similarly,

$$
\gamma^{2} \xi^{2}(a v, v) \leq \gamma^{2} \xi^{2}\|a\|_{\infty}\|v\|^{2}=C \gamma^{2} \xi^{2}\|v\|^{2},
$$

and, using Poincaré's inequality $\|v\| \leq\left\|v^{\prime}\right\|$,

$$
\gamma \xi|(b s(\cdot-y) v, v)| \leq \gamma \xi\|b\|_{\infty}\left\|v^{\prime}\right\|\|v\| \leq \frac{1}{4}\left\|v^{\prime}\right\|^{2}+C \gamma^{2} \xi^{2}\|v\|^{2} .
$$

Thus the right-hand side of (2.13) can be bounded by

$$
\frac{1}{2} \gamma^{-1} \xi^{-1}+\frac{3}{4}\left\|v^{\prime}\right\|^{2}+C \gamma^{2} \xi^{2}\|v\|^{2}
$$

and Lemma 2.1 yields

$$
\frac{1}{4}\left\|v^{\prime}\right\|^{2}+\left(1-C \gamma^{2}\right) \xi^{2}\|v\|^{2} \leq \frac{1}{2} \gamma^{-1} \xi^{-1}, \quad \lambda \in \Sigma_{\kappa, \theta} .
$$

By taking $\gamma$ sufficiently small, we arrive at

$$
\|v\| \leq C \xi^{-3 / 2}, \quad\left\|v^{\prime}\right\| \leq C \xi^{-1 / 2}, \quad \lambda \in \Sigma_{\kappa, \theta},
$$

which in view of (2.14) implies the desired bound (2.11).

Remark. Since $\|g(\cdot, y, \lambda)\|_{1}=\left\|v(\cdot, y, \lambda) e^{-\gamma \xi|\cdot-y|}\right\|_{1} \leq C \xi^{-1 / 2}\|v(\cdot, y, \lambda)\|$, we note that (2.9) actually follows directly from (2.16) without passing through (2.11).

We now turn to the corresponding result for the discrete problem (1.3).

Theorem 2.3. For any $\theta \in\left(\frac{1}{2} \pi, \pi\right)$ there are $\kappa \geq 0$ and $C \geq 1$ such that

$$
\left\|R\left(\lambda,-A_{h}\right) P_{h} f\right\|_{p} \leq \frac{C}{|\lambda-\kappa|}\|f\|_{p}, \quad f \in L_{p}(\Omega), \lambda \in \Sigma_{\kappa, \theta}, \quad 1 \leq p \leq \infty .
$$

Proof. Let $\theta \in\left(\frac{1}{2} \pi, \pi\right)$ be arbitrary and let $\kappa$ be as in Lemma 2.1. Referring to the discussion at the beginning of the proof of Theorem 2.2, we note that it suffices to show the resolvent estimate for $p=\infty$.

Step 1. We first dispose of the case when $\lambda$ is large compared to $h^{-2}$ by noting that

$$
\left\|A_{h} \chi\right\|_{\infty} \leq C_{1} h^{-2}\|\chi\|_{\infty}, \quad \chi \in S_{h} .
$$

In fact, by inverse inequalities and the stability of $P_{h}$ in $L_{1}(\Omega)$ (see [4]), we have

$$
\begin{aligned}
\left(A_{h} \chi, \phi\right) & =\left(A_{h} \chi, P_{h} \phi\right)=A\left(\chi, P_{h} \phi\right) \leq C\left\|\chi^{\prime}\right\|_{\infty}\left\|\left(P_{h} \phi\right)^{\prime}\right\|_{1} \\
& \leq C h^{-2}\|\chi\|_{\infty}\left\|P_{h} \phi\right\|_{1} \leq C_{1} h^{-2}\|\chi\|_{\infty}\|\phi\|_{1},
\end{aligned}
$$


for $\chi \in S_{h}, \phi \in L_{1}(\Omega)$, which shows (2.17). Thus, if

$$
|\lambda| \geq C_{2} h^{-2}, \quad \text { where } C_{2}=\max \left(2 C_{1}, \kappa\right),
$$

then $\left\|\lambda^{-1} A_{h} \chi\right\|_{\infty} \leq \frac{1}{2}\|\chi\|_{\infty}$, so that by the stability of $P_{h}$ in $L_{\infty}(\Omega)$ (see [4])

$$
\begin{aligned}
\left\|\left(\lambda I+A_{h}\right)^{-1} P_{h} f\right\|_{\infty} & =\left\|\lambda^{-1} \sum_{j=0}^{\infty}\left(-\lambda^{-1} A_{h}\right)^{j} P_{h} f\right\|_{\infty} \\
& \leq \frac{2}{|\lambda|}\left\|P_{h} f\right\|_{\infty} \leq \frac{C}{|\lambda|}\|f\|_{\infty} .
\end{aligned}
$$

Since (2.18) implies (note that $h \leq 1$ )

$$
\kappa \leq C_{2} \leq C_{2} h^{-2}
$$

so that $|\lambda-\kappa| \leq 2|\lambda|$, we may conclude that the desired estimate holds for $|\lambda| \geq C_{2} h^{-2}$.

Step 2. In this step of the proof we shall bound the solution of (1.3) at the nodal points and show

$$
\max _{1 \leq j \leq N}\left|u_{h}\left(x_{j}, \lambda\right)\right| \leq \frac{C}{|\lambda-\kappa|}\|f\|_{\infty}, \quad \lambda \in \Sigma_{\kappa, \theta}, \quad|\lambda| \leq C_{2} h^{-2}
$$

For future reference we note that, by (2.3) and (2.20), the assumption $|\lambda| \leq$ $C_{2} h^{-2}$ implies that $\xi^{2} \leq|\lambda-\kappa| \leq|\lambda|+\kappa \leq 2 C_{2} h^{-2}$, that is, $\xi h \leq C$.

Following the proof of Theorem 2.2, we let $x_{j}$ be an arbitrary nodal point and introduce the discrete Green's function $g_{h}=g_{h}\left(\cdot, x_{j}, \lambda\right) \in S_{h}$ defined by

$$
A\left(\chi_{h}, g_{h}\right)+\lambda\left(\chi_{h}, g_{h}\right)=\chi_{h}\left(x_{j}\right), \quad \forall \chi_{h} \in S_{h} .
$$

With $v(x)=v\left(x, x_{j}, \lambda\right)=g_{h}\left(x, x_{j}, \lambda\right) e^{\gamma \xi\left|x-x_{j}\right|}$, we shall show for some $\gamma \in$ $(0,1)$ that

$$
\left\|v\left(\cdot, x_{j}, \lambda\right)\right\|_{\infty} \leq C \xi^{-1}, \quad j=1, \ldots, N, \lambda \in \Sigma_{\kappa, \theta},|\lambda| \leq C_{2} h^{-2},
$$

which leads to (2.21) in the same way as in the proof of Theorem 2.2.

With $\chi(x)=v(x) e^{\gamma \xi\left|x-x_{j}\right|}$, the argument leading to (2.13) now yields

$$
\begin{aligned}
A(v, v)+\lambda\|v\|^{2}= & A\left(\chi-\chi_{h}, g_{h}\right)+\lambda\left(\chi-\chi_{h}, g_{h}\right) \\
& +\chi_{h}\left(x_{j}\right)+2 i \gamma \xi \operatorname{Im}\left(a s\left(\cdot-x_{j}\right) v^{\prime}, v\right) \\
& +\gamma^{2} \xi^{2}(a v, v)-\gamma \xi\left(b s\left(\cdot-x_{j}\right) v, v\right),
\end{aligned}
$$

where $\chi_{h} \in S_{h}$ is arbitrary. For $\chi_{h}$ we choose an "elliptic projection" of $\chi$ defined by

$$
\left(\chi_{h}^{\prime}, \phi^{\prime}\right)=\left(\chi^{\prime}, \phi^{\prime}\right), \quad \forall \phi \in S_{h} .
$$

It is well known that $\chi_{h}\left(x_{j}\right)=\chi\left(x_{j}\right)$, so that $\chi_{h}\left(x_{j}\right)=v\left(x_{j}\right)$. The last four terms are thus the same as in (2.13) and can be bounded as in (2.15). For the two remaining terms we have, by a well-known error estimate and an inverse 
inequality, with $\|u\|_{\Omega_{i}}^{2}=\int_{\Omega_{i}}|u|^{2} d x$,

$$
\begin{aligned}
& \left|A\left(\chi-\chi_{h}, g_{h}\right)+\lambda\left(\chi-\chi_{h}, g_{h}\right)\right| \\
& \leq C \sum_{i=0}^{N}\left(\left\|\chi^{\prime}-\chi_{h}^{\prime}\right\|_{\Omega_{i}}\left\|g_{h}^{\prime}\right\|_{\Omega_{i}}+|\lambda|\left\|\chi-\chi_{h}\right\|_{\Omega_{i}}\left\|g_{h}\right\|_{\Omega_{i}}\right) \\
& \leq C \sum_{i=0}^{N}\left(h_{i}^{r-1}\left\|\chi^{(r)}\right\|_{\Omega_{i}}\left\|g_{h}^{\prime}\right\|_{\Omega_{i}}+|\lambda| h_{i}^{r}\left\|\chi^{(r)}\right\|_{\Omega_{i}}\left\|g_{h}\right\|_{\Omega_{i}}\right) \\
& \quad \leq C \sum_{i=0}^{N} h_{i}^{r-2}\left\|\chi^{(r)}\right\|_{\Omega_{i}}\left\|g_{h}\right\|_{\Omega_{i}},
\end{aligned}
$$

where we have also used the assumption that $|\lambda| \leq C_{2} h^{-2}$. Recalling that $\chi(x)=g_{h}(x) e^{2 \gamma \xi\left|x-x_{j}\right|}$, where $g_{h}^{(r)}(x) \equiv 0$ on $\Omega_{i}$, and that $\xi h \leq C$ and $\gamma \in(0,1)$, we have

$$
\begin{aligned}
h_{i}^{r-2}\left\|\chi^{(r)}\right\|_{\Omega_{i}} & \leq C e^{C \gamma} h_{i}^{r-2} \sum_{l=0}^{r-1}(\gamma \xi)^{r-l}\left\|g_{h}^{(l)}\right\|_{\Omega_{i}} e^{2 \gamma \xi\left|x_{i}-x_{j}\right|} \\
& \leq C h_{i}^{r-2}\left((\gamma \xi)^{r}\left\|g_{h}\right\|_{\Omega_{i}}+\sum_{l=1}^{r-1}(\gamma \xi)^{r-l} h_{i}^{1-l}\left\|g_{h}^{\prime}\right\|_{\Omega_{i}}\right) e^{2 \gamma \xi\left|x_{i}-x_{j}\right|} \\
& \leq C\left(\gamma \xi\left\|g_{h}\right\|_{\Omega_{i}}+\left\|g_{h}^{\prime}\right\|_{\Omega_{i}}\right) \gamma \xi e^{2 \gamma \xi\left|x_{i}-x_{j}\right|}
\end{aligned}
$$

Hence,

$$
h_{i}^{r-2}\left\|\chi^{(r)}\right\|_{\Omega_{i}}\left\|g_{h}\right\|_{\Omega_{i}} \leq C\left(\left\|g_{h}^{\prime}\right\|_{\Omega_{i}}+\gamma \xi\left\|g_{h}\right\|_{\Omega_{i}}\right) \gamma \xi\left\|g_{h}\right\|_{\Omega_{i}} e^{2 \gamma \xi\left|x_{i}-x_{j}\right|},
$$

and since, cf. (2.12),

$$
\left\|g_{h}^{\prime}\right\|_{\Omega_{i}}+\gamma \xi\left\|g_{h}\right\|_{\Omega_{i}} \leq C\left(\left\|v^{\prime}\right\|_{\Omega_{i}}+\gamma \xi\|v\|_{\Omega_{i}}\right) e^{-\gamma \xi\left|x_{i}-x_{j}\right|},
$$

we may finally conclude that

$$
\left|A\left(\chi-\chi_{h}, g_{h}\right)+\lambda\left(\chi-\chi_{h}, g_{h}\right)\right| \leq \frac{1}{8}\left\|v^{\prime}\right\|^{2}+C \gamma^{2} \xi^{2}\|v\|^{2} .
$$

Thus, the right-hand side of (2.23) may be bounded as in (2.15) and the bound (2.21) follows for $\gamma$ sufficiently small by application of Lemma 2.1 as in the proof of Theorem 2.2.

Step 3. Having established the estimate (2.21) for $u_{h}$ at the nodal points, we now want to show the same bound in the interiors of the subintervals. (Of course this is trivial if $r=2$.) Assume that the maximum of $\left|u_{h}\right|$ is attained in the subinterval $\Omega_{l}$. We introduce the subspace

$$
S_{h}\left(\Omega_{l}\right)=\left\{\chi \in S_{h}: \operatorname{supp} \chi \subset \bar{\Omega}_{l}\right\},
$$

and define $v_{h} \in S_{h}\left(\Omega_{l}\right)$ by

$$
A\left(v_{h}-u_{h}, \chi\right)+\kappa\left(v_{h}-u_{h}, \chi\right)=0, \quad \forall \chi \in S_{h}\left(\Omega_{l}\right) .
$$

We shall first show for $\tilde{u}_{h}=u_{h}-v_{h} \in S_{h}$ that with $\|u\|_{\infty, \Omega_{l}}=\sup _{\Omega_{l}}|u|$,

$$
\left\|\tilde{u}_{h}\right\|_{\infty, \Omega_{l}} \leq C \max \left(\left|u_{h}\left(x_{l}\right)\right|,\left|u_{h}\left(x_{l+1}\right)\right|\right),
$$


which in view of (2.21) implies

$$
\left\|\tilde{u}_{h}\right\|_{\infty, \Omega_{l}} \leq \frac{C}{|\lambda-\kappa|}\|f\|_{\infty}, \quad \lambda \in \Sigma_{\kappa, \theta}, \quad|\lambda| \leq C_{2} h^{-2} .
$$

We shall then show

$$
\left\|v_{h}\right\|_{\infty} \leq \frac{C}{|\lambda-\kappa|}\|f\|_{\infty}, \quad \lambda \in \Sigma_{\kappa, \theta}, \quad|\lambda| \leq C_{2} h^{-2},
$$

which completes the proof, since $u_{h}=\tilde{u}_{h}+v_{h}$.

To show (2.25) we note that, with $\hat{u}_{h}$ equal to the linear interpolant of $u_{h}$ on $\Omega_{l}$ and $\hat{u}_{h}=u_{h}$ elsewhere, equation (2.24) implies

$$
A\left(\hat{u}_{h}-\tilde{u}_{h}, \chi\right)+\kappa\left(\hat{u}_{h}-\tilde{u}_{h}, \chi\right)=A\left(\hat{u}_{h}, \chi\right)+\kappa\left(\hat{u}_{h}, \chi\right), \quad \forall \chi \in S_{h}\left(\Omega_{l}\right) .
$$

Since $w_{h}=\hat{u}_{h}-\tilde{u}_{h} \in S_{h}\left(\Omega_{l}\right)$, this implies

$$
A\left(w_{h}, w_{h}\right)+\kappa\left\|w_{h}\right\|^{2}=A\left(\hat{u}_{h}, w_{h}\right)+\kappa\left(\hat{u}_{h}, w_{h}\right),
$$

and an application of Lemma 2.1 (with $\lambda=\kappa$ and with $\|u\|_{\Omega_{l}}^{2}=\int_{\Omega_{l}}|u|^{2} d x$ ) shows

$$
\left\|w_{h}^{\prime}\right\|^{2} \leq C\left|A\left(\hat{u}_{h}, w_{h}\right)+\kappa\left(\hat{u}_{h}, w_{h}\right)\right| \leq C\left\|\hat{u}_{h}^{\prime}\right\|_{\Omega_{l}}^{2}+C\left\|\hat{u}_{h}\right\|_{\Omega_{l}}^{2}+\frac{1}{2}\left\|w_{h}^{\prime}\right\|^{2} .
$$

Since

$\left\|w_{h}\right\|_{\infty} \leq h^{1 / 2}\left\|w_{h}^{\prime}\right\|, \quad h^{-1 / 2}\left\|\hat{u}_{h}\right\|_{\Omega_{l}}+h^{1 / 2}\left\|\hat{u}_{h}^{\prime}\right\|_{\Omega_{l}} \leq C \max \left(\left|u_{h}\left(x_{l}\right)\right|,\left|u_{h}\left(x_{l+1}\right)\right|\right)$, we may conclude that

$$
\left\|w_{h}\right\|_{\infty} \leq C \max \left(\left|u_{h}\left(x_{l}\right)\right|,\left|u_{h}\left(x_{l+1}\right)\right|\right),
$$

and (2.25) follows, since $\tilde{u}_{h}=\hat{u}_{h}-w_{h}$ and $\left\|\hat{u}_{h}\right\|_{\infty, \Omega_{l}} \leq \max \left(\left|u_{h}\left(x_{l}\right)\right|,\left|u_{h}\left(x_{l+1}\right)\right|\right)$.

It remains to estimate $\left\|v_{h}\right\|_{\infty}$. From (2.24) and (1.3) we have

$$
A\left(v_{h}, \chi\right)+\lambda\left(v_{h}, \chi\right)=(f, \chi)-(\lambda-\kappa)\left(\tilde{u}_{h}, \chi\right), \quad \forall \chi \in S_{h}\left(\Omega_{l}\right) .
$$

Taking $\chi=v_{h}$, we obtain

$$
A\left(v_{h}, v_{h}\right)+\lambda\left\|v_{h}\right\|^{2}=\left(f, v_{h}\right)-(\lambda-\kappa)\left(\tilde{u}_{h}, v_{h}\right) .
$$

An application of Lemma 2.1 yields, in view of (2.3) and (2.26),

$$
\begin{aligned}
\left\|v_{h}^{\prime}\right\|^{2}+\xi^{2}\left\|v_{h}\right\|^{2} & \leq C\left|\left(f, v_{h}\right)-(\lambda-\kappa)\left(\tilde{u}_{h}, v_{h}\right)\right| \\
& \leq C\left(\|f\|_{\infty}+|\lambda-\kappa|\left\|\tilde{u}_{h}\right\|_{\infty, \Omega_{l}}\right)\left\|v_{h}\right\|_{1} \\
& \leq C h^{1 / 2}\|f\|_{\infty}\left\|v_{h}\right\| .
\end{aligned}
$$

Hence,

$$
\left\|v_{h}\right\| \leq C h^{1 / 2} \xi^{-2}\|f\|_{\infty} .
$$

Since $\left\|v_{h}\right\|_{\infty} \leq C h^{-1 / 2}\left\|v_{h}\right\|$ by an inverse inequality, we conclude that (2.27) holds.

We may now prove our main result.

Proof of Theorem 1.1. We only consider $R(\lambda,-A)$. The proof for $R\left(\lambda,-A_{h}\right)$ is identical. Fix $\theta \in\left(\frac{1}{2} \pi, \pi\right)$. By Theorem 2.2 there are $\kappa \geq 0$ and $C \geq 1$ such that

$$
\|R(\lambda,-A) f\|_{p} \leq \frac{C}{|\lambda-\kappa|}\|f\|_{p}, \quad \lambda \in \Sigma_{\kappa, \theta} .
$$


The assumption (1.1) and Lemma 2.1 imply that $B_{\lambda}(u, v)=A(u, v)+\lambda(u, v)$ is coercive uniformly for $\lambda \in \Sigma_{-a_{0} / 2, \pi / 2} \cup \Sigma_{\kappa, \theta}$, so that, by Lax-Milgram's lemma, equation (1.2) has a unique solution and

$$
\|R(\lambda,-A) f\|_{H_{0}^{1}} \leq C\|f\|_{H^{-1}}, \quad f \in H^{-1}(\Omega), \lambda \in \Sigma_{-a_{0} / 2, \pi / 2} \cup \Sigma_{\kappa, \theta} .
$$

Hence, $\|R(\lambda,-A) f\|_{p} \leq C\|f\|_{p}$, and using also (2.29), we get

$$
\|R(\lambda,-A) f\|_{p} \leq \frac{C}{1+|\lambda|}\|f\|_{p}, \quad \lambda \in \Sigma_{-a_{0} / 2, \pi / 2} \cup \Sigma_{\kappa, \theta} .
$$

We then choose $\varphi \in\left(\frac{1}{2} \pi, \theta\right]$ such that $\Sigma_{0, \varphi} \subset \Sigma_{-a_{0} / 2, \pi / 2} \cup \Sigma_{\kappa, \theta}$. Together, these estimates show the required bounds for $R(\lambda,-A)$ for $\lambda \in \Sigma_{\varphi}=\Sigma_{0, \varphi}$.

We shall also need an estimate of the norms of the resolvents considered as operators from $\mathscr{M}(\Omega)$ to $L_{\infty}(\Omega)$, where $\mathscr{M}(\Omega)$ denotes the set of finite measures $\mu$ with $\|\mu\|_{\mathscr{M}}=|\mu|(\Omega)=\int_{\Omega} d|\mu|$. With each $\mu \in \mathscr{M}(\Omega)$ we associate a distribution (also denoted $\mu$ ) in the usual way: $\langle\mu, \chi\rangle=\int_{\Omega} \chi(x) d \mu(x)$ for $\chi \in C(\bar{\Omega})$. Recall that $P_{h}: \mathscr{M}(\Omega) \rightarrow S_{h}$ is defined in (1.4).

Theorem 2.4. There are $\varphi \in\left(\frac{1}{2} \pi, \pi\right)$ and $C \geq 1$ such that

$\|R(\lambda,-A) f\|_{\infty}+\left\|R\left(\lambda,-A_{h}\right) P_{h} f\right\|_{\infty} \leq \frac{C}{\sqrt{1+|\lambda|}}\|f\|_{\mathscr{M}}, \quad f \in \mathscr{M}(\Omega), \lambda \in \Sigma_{\varphi}$.

Proof. Let $\theta \in\left(\frac{1}{2} \pi, \pi\right)$ be arbitrary, and let $\kappa$ be as in Lemma 2.1. Taking $\chi=u$ in (1.2) and applying the lemma shows

$$
\left\|u^{\prime}\right\|^{2}+\xi^{2}\|u\|^{2} \leq C|\langle f, \bar{u}\rangle| \leq C\|f\|_{\mathscr{M}}\|u\|_{\infty}, \quad \lambda \in \Sigma_{\kappa, \theta},
$$

which in view of (2.14) and the arguments in the proof of Theorem 1.1 implies the desired result for $R(\lambda,-A)$. The same argument applies to $R\left(\lambda,-A_{h}\right)$.

\section{ERROR ESTIMATES}

In view of the assumption (1.1) and the Lax-Milgram lemma we may define a bounded linear operator $R_{h}: H_{0}^{1}(\Omega) \rightarrow S_{h}$ by the equation

$$
A\left(R_{h} v-v, \chi\right)=0, \quad \forall \chi \in S_{h} .
$$

For this "Ritz projection" we have the following error estimates.

Lemma 3.1. There is a constant $C$ such that for $k=1, \ldots, r, 1 \leq p \leq \infty$, we have

$$
\left\|R_{h} v-v\right\|_{p}+h\left\|\left(R_{h} v-v\right)^{\prime}\right\|_{p} \leq C h^{k}\|v\|_{k, p}, \quad v \in H_{0}^{1}(\Omega) \cap W_{p}^{k}(\Omega) .
$$

Proof. The case $p=\infty$ can be found in [14] or [7]. We prove the case $p=1$ by a slight modification of the argument of [7]. The remaining case $1<p<\infty$ then follows by interpolation.

We shall show below that

$$
\left\|\left(R_{h} v-v\right)^{\prime}\right\|_{1} \leq C h^{k-1}\|v\|_{k, 1}, \quad k=1, \ldots, r .
$$

The desired bound for $\left\|R_{h} v-v\right\|_{1}$ then follows by the standard duality argument. In fact, let $\phi \in L_{\infty}(\Omega)$ be arbitrary and define $\psi \in H_{0}^{1}(\Omega)$ by

$$
A(\chi, \psi)=(\chi, \phi), \quad \forall \chi \in H_{0}^{1}(\Omega) .
$$


Then

$$
\begin{aligned}
\left(R_{h} v-v, \phi\right) & =A\left(R_{h} v-v, \psi\right)=A\left(R_{h} v-v, \psi-R_{h} \psi\right) \\
& \leq C\left\|\left(R_{h} v-v\right)^{\prime}\right\|_{1}\left\|\left(R_{h} \psi-\psi\right)^{\prime}\right\|_{\infty} \\
& \leq C h^{k-1}\|v\|_{k, 1} C h\|\psi\|_{2, \infty},
\end{aligned}
$$

which implies $\left\|R_{h} v-v\right\|_{1} \leq C h^{k}\|v\|_{k, 1}$, since clearly $\|\psi\|_{2, \infty} \leq C\|\phi\|_{\infty}$. by

In order to show (3.2), we introduce the projection $\tilde{R}_{h}: H_{0}^{1}(\Omega) \rightarrow S_{h}$ defined

$$
\left(\left(\tilde{R}_{h} v-v\right)^{\prime}, \chi^{\prime}\right)=0, \quad \forall \chi \in S_{h} .
$$

It follows that $\left(\tilde{R}_{h} v\right)^{\prime}=\tilde{P}_{h} v^{\prime}$, where $\tilde{P}_{h}$ denotes the $L_{2}$ projection onto $V_{h}=$ $\left\{\chi \in L_{2}(\Omega):\left.\chi\right|_{\Omega_{i}} \in \Pi_{r-2}\right\}$, see $[7, \S 4]$. Hence,

$$
\left\|\left(\tilde{R}_{h} v-v\right)^{\prime}\right\|_{1} \leq C h^{k-1}\|v\|_{k, 1}, \quad k=1, \ldots, r,
$$

since $\tilde{P}_{h}$ is stable in $L_{1}(\dot{\Omega})$ (this is trivial because $\tilde{P}_{h}$ is a local projection).

It remains to estimate $\theta=R_{h} v-\tilde{R}_{h} v$. We introduce the piecewise constant function $\tilde{a}$ defined by $\tilde{a}(x)=a\left(x_{k+1 / 2}\right)$ for $x \in \Omega_{k}$, where $x_{k+1 / 2}$ denotes the midpoint of $\Omega_{k}$, and we note that

$$
\left(\tilde{a}\left(v-\tilde{R}_{h} v\right)^{\prime}, \chi^{\prime}\right)_{\Omega_{k}}=0, \quad \forall \chi \in S_{h} .
$$

Since $\theta \in S_{h}$, we thus have

$$
\begin{aligned}
a_{0}\left\|\theta^{\prime}\right\|_{2}^{2} & \leq A(\theta, \theta)=A\left(R_{h} v-\tilde{R}_{h} v, \theta\right)=A\left(v-\tilde{R}_{h} v, \theta\right) \\
& =\left(a\left(v-\tilde{R}_{h} v\right)^{\prime}, \theta^{\prime}\right)+\left(b\left(v-\tilde{R}_{h} v\right)^{\prime}, \theta\right)+\left(c\left(v-\tilde{R}_{h} v\right), \theta\right) \\
& =\left((a-\tilde{a})\left(v-\tilde{R}_{h} v\right)^{\prime}, \theta^{\prime}\right)+\left(b\left(v-\tilde{R}_{h} v\right)^{\prime}, \theta\right)+\left(c\left(v-\tilde{R}_{h} v\right), \theta\right) .
\end{aligned}
$$

Hence,

$\left\|\theta^{\prime}\right\|_{2}^{2} \leq C h\left\|\left(v-\tilde{R}_{h} v\right)^{\prime}\right\|_{1}\left\|\theta^{\prime}\right\|_{\infty}+C\left\|\left(v-\tilde{R}_{h} v\right)^{\prime}\right\|_{1}\|\theta\|_{\infty} \leq C\left\|\left(v-\tilde{R}_{h} v\right)^{\prime}\right\|_{1}\left\|^{\prime}\right\|_{2}$.

Therefore,

$$
\left\|\theta^{\prime}\right\|_{1} \leq\left\|\theta^{\prime}\right\|_{2} \leq C\left\|\left(v-\tilde{R}_{h} v\right)^{\prime}\right\|_{1},
$$

which together with (3.3) implies (3.2).

We now turn to the proof of an error estimate in the case of smooth solutions of the nonhomogeneous problem.

Theorem 3.2. Let $1 \leq p \leq \infty$, let $u$ be a sufficiently smooth solution of (1.5) with $u_{0} \in H_{0}^{1}(\Omega) \cap W_{p}^{r}(\Omega)$, and let $u_{h}$ be the corresponding solution of (1.6). Then

$$
\left\|u_{h}(t)-u(t)\right\|_{p} \leq C\left\|u_{0 h}-u_{0}\right\|_{p}+C h^{r}\left(\left\|u_{0}\right\|_{r, p}+\int_{0}^{t}\left\|u_{t}\right\|_{r, p} d s\right) .
$$

Proof. Following a standard practice, we divide the error into two parts:

$$
e(t) \equiv u_{h}(t)-u(t)=\left(u_{h}(t)-R_{h} u(t)\right)+\left(R_{h} u(t)-u(t)\right) \equiv \theta(t)+\rho(t)
$$

In view of Lemma 3.1, we have

$$
\left\|D_{t}^{l} \rho(t)\right\|_{p} \leq C h^{k}\left\|D_{t}^{l} u(t)\right\|_{k, p}, \quad l=0,1, k=1, \ldots, r
$$


where $D_{t}=\partial / \partial t$. Since this estimates $\|\rho(t)\|_{p}$ in the appropriate way, it only remains to estimate $\theta(t)$, which belongs to $S_{h}$. In view of (1.6), the identity $A_{h} R_{h}=P_{h} A$ and (1.5), we find that $\theta_{t}+A_{h} \theta=-P_{h} \rho_{t}$, and hence

$$
\theta(t)=E_{h}(t) \theta(0)-\int_{0}^{t} E_{h}(t-s) P_{h} \rho_{s}(s) d s .
$$

The desired error bound now follows immediately by application of (3.5) and the stability estimate for $E_{h}(t)$ in (1.11).

The proof of Theorem 3.2 is based on the stability in $L_{p}(\Omega)$ of the discrete evolution operator $E_{h}(t)$ together with the "truncation error estimate" of Lemma 3.1. Since $L_{p}(\Omega)$ stability is also available for completely discrete evolution operators $E_{k h}^{n}=r\left(-k A_{h}\right)^{n}$ based on $A$-acceptable rational functions, see (1.17), it is possible to obtain analogous error estimates for certain completely discrete schemes. We carry this out for the backward Euler and Crank-Nicolson methods in the following theorem.

Theorem 3.3. Let $1 \leq p \leq \infty$, let $u$ be a sufficiently smooth solution of (1.5) with $u_{0} \in H_{0}^{1}(\Omega) \cap W_{p}^{r}(\Omega)$, and let $U_{n}$ be the corresponding approximation obtained by the backward Euler method (1.18). Then

$$
\left\|U_{n}-u\left(t_{n}\right)\right\|_{p} \leq C\left\|u_{0 h}-u_{0}\right\|_{p}+C h^{r}\left(\left\|u_{0}\right\|_{r, p}+\int_{0}^{t_{n}}\left\|u_{t}\right\|_{r, p} d s\right)+C k \int_{0}^{t_{n}}\left\|u_{t t}\right\|_{p} d s .
$$

For the Crank-Nicolson method (1.19) we have

$$
\begin{aligned}
\left\|U_{n}-u\left(t_{n}\right)\right\|_{p} \leq & C\left\|u_{0 h}-u_{0}\right\|_{p}+C h^{r}\left(\left\|u_{0}\right\|_{r, p}+\int_{0}^{t_{n}}\left\|u_{t}\right\|_{r, p} d s\right) \\
& +C k^{2} \int_{0}^{t_{n}}\left(\left\|u_{t t t}\right\|_{p}+\left\|u_{t t}\right\|_{2, p}\right) d s .
\end{aligned}
$$

Proof. Using a splitting of the error analogous to (3.4), we have for the backward Euler method instead of (3.6)

$$
\theta_{n}=E_{k h}^{n} \theta_{0}-k \sum_{j=1}^{n} E_{k h}^{n-j+1} P_{h}\left(\bar{\partial}_{t} \rho_{j}+\left(\bar{\partial}_{t} u\left(t_{j}\right)-u_{t}\left(t_{j}\right)\right)\right),
$$

where $E_{k h}=\left(I+k A_{h}\right)^{-1}$, from which the proof proceeds using the stability property $(1.17)$ with $r(\lambda)=1 /(1-\lambda)$ and standard estimates. The argument for the Crank-Nicolson argument is analogous.

General time-discretization schemes require more complicated approximations of the source term $f$; see [2]. Avoiding this difficulty, we shall content ourselves in the rest of this section with studying the homogeneous equation $(f \equiv 0)$. We will then need smoothing properties, which are slightly more general than (1.10). In particular, we need to deal with solutions which already possess some initial smoothness and compatibility. In order to express this we define for nonnegative integers $m$ and for all $p \in[1, \infty]$

$\grave{W}_{p}^{m}(\Omega)=\left\{v \in W_{p}^{m}(\Omega): A^{j} v=0\right.$ at $x=0,1$ for all integers $\left.j \in[0, m / 2)\right\}$. 
Lemma 3.4. Let $m$ and $k$ be nonnegative integers. Then

$$
\begin{aligned}
\|E(t) v\|_{m, p}+t\left\|E^{\prime}(t) v\right\|_{m, p} & \leq C_{m} t^{-m / 2}\|v\|_{p}, & & t>0, v \in L_{p}(\Omega), \\
\|E(t) v\|_{\infty}+\left\|E_{h}(t) P_{h} v\right\|_{\infty} & \leq C t^{-1 / 2}\|v\|_{\mathscr{M}}, & & t>0, v \in \mathscr{M}(\Omega) . \\
\left\|A^{k} E(t) v\right\|_{m, p} & \leq C_{k, m} t^{-k}\|v\|_{m, p}, & & t>0, v \in \dot{W}_{p}^{m}(\Omega) .
\end{aligned}
$$

Proof. The regularity estimate (3.7) follows from the analyticity of $E(t)$, see (1.10), together with (1.7) and the interpolation inequality

$$
\left\|u^{\prime}\right\|_{p} \leq C \sqrt{\|u\|_{p}\left\|u^{\prime \prime}\right\|_{p}}, \quad 1 \leq p \leq \infty, u \in \dot{W}_{p}^{2}(\Omega),
$$

which can be proved by elementary arguments. In fact, let $m=2 l+i$ with $l \geq 0$ and $i=0$ or 1 . Then

$$
\begin{aligned}
\|E(t) v\|_{m, p} & \leq C_{m}\left\|A^{l} E(t) v\right\|_{i, p} \leq C_{m} \sqrt{\left\|A^{l} E(t) v\right\|_{0, p}\left\|A^{l} E(t) v\right\|_{2 i, p}} \\
& \leq C_{m} \sqrt{\left\|A^{l} E(t) v\right\|_{0, p}\left\|A^{l+i} E(t) v\right\|_{0, p}} \leq C_{m} t^{-m / 2}\|v\|_{p},
\end{aligned}
$$

and similarly for $E^{\prime}(t) v$. The bounds in (3.8) follow from the resolvent estimates of Theorem 2.4 and the contour integral representation of the semigroups in the same way as (1.10), (1.11).

We now turn to the proof of (3.9) and first note that it suffices to consider the cases $k=0$ and 1 , since the case $k \geq 2$ follows from these and the identity $A^{k} E(t) v=(A E(t / k))^{k} v$. We also note that the case $m=0$ follows from (1.10). Assume now that (3.9) has been proved also for $m=1$. Then for $m \geq 2$ we may write $m=2 l+i$ with $l \geq 0, i=0$ or 1 , and obtain for $k=0,1$

$$
\begin{aligned}
\left\|A^{k} E(t) v\right\|_{m, p} & \leq C\left\|A^{k+l} E(t) v\right\|_{i, p}=C\left\|A^{k} E(t) A^{l} v\right\|_{i, p} \\
& \leq C t^{-k}\left\|A^{l} v\right\|_{i, p} \leq C_{m} t^{-k}\|v\|_{m, p}, \quad v \in \dot{W}_{p}^{m}(\Omega) .
\end{aligned}
$$

It remains to consider $m=1$. For $k=1$ we have

$$
\begin{aligned}
\|A E(t) v\|_{1, p} & \leq C \sqrt{\left\|A^{2} E(t) v\right\|_{p}\|A E(t) v\|_{p}} \\
& \leq C t^{i-3 / 2}\|v\|_{2 i, p}, \quad v \in \dot{W}_{p}^{2 i}(\Omega), \quad i=0,1,
\end{aligned}
$$

from which the desired result follows by interpolation. In fact, for each $v \in$ $\dot{W}_{p}^{1}(\Omega)$ and $\epsilon>0$ there is $v_{\epsilon} \in \dot{W}_{p}^{2}(\Omega)$ such that $\left\|v-v_{\epsilon}\right\|_{p} \leq C \epsilon\|v\|_{1, p}$ and $\left\|v_{\epsilon}\right\|_{2, p} \leq C \epsilon^{-1}\|v\|_{1, p}$. For $\epsilon \leq 1$ this is achieved in a standard way by extension of $v$ to an odd 2-periodic function and by convolution with an even mollifier on the scale $\epsilon$. If $\epsilon>1$, then we take $v_{\epsilon}=0$. Hence with $\epsilon=t^{1 / 2}$ we obtain

$$
\begin{aligned}
\|A E(t) v\|_{1, p} & \leq\left\|A E(t)\left(v-v_{\epsilon}\right)\right\|_{1, p}+\left\|A E(t) v_{\epsilon}\right\|_{1, p} \\
& \leq C t^{-3 / 2}\left\|v-v_{\epsilon}\right\|_{p}+C t^{-1 / 2}\left\|v_{\epsilon}\right\|_{2, p} \leq C t^{-1}\|v\|_{1, p}, \quad v \in \dot{W}_{p}^{1}(\Omega) .
\end{aligned}
$$

The final case $m=1, k=0$ follows from the Cauchy integral representation (1.8) once we have shown the resolvent estimate

$$
\|R(\lambda,-A) f\|_{1, p} \leq \frac{C}{1+|\lambda|}\|f\|_{1, p}, \quad f \in \stackrel{\circ}{W}_{p}^{1}(\Omega) .
$$


To prove this, we set $u=R(\lambda,-A) f$ and $v=a B u^{\prime}$, where $B(x)=$ $\exp \left(-\int_{0}^{x} \frac{b(t)}{a(t)} d t\right)$. Then it is easy to show that $v \in W_{p}^{2}(\Omega)$ and $v^{\prime}=$ $B(\lambda u+c u-f)$, so that

$$
-\left(a v^{\prime}\right)^{\prime}+c v+\lambda v=g, \quad x \in \Omega ; \quad v^{\prime}(0)=v^{\prime}(1)=0,
$$

where $g=(a B f)^{\prime}-(a B)^{\prime} \lambda u-(c a B)^{\prime} u$ satisfies $\|g\|_{p} \leq C\|f\|_{1, p}$. An argument similar to the proof of Theorem 1.1 now gives $\|v\|_{p} \leq \frac{C}{1+|\lambda|}\|g\|_{p}$, which implies (3.10). The proof of Lemma 3.4 is now complete.

Remark. The interpolation argument that we used in the proof of the special case $m=1, k=1$ of (3.9) may also be expressed by saying that $\dot{W}_{p}^{1}(\Omega)$ is continuously imbedded in the space $\left(L_{p}(\Omega), \mathscr{W}_{p}^{2}(\Omega)\right)_{1 / 2, \infty}$ defined by the real interpolation method. The estimate (3.9) follows more directly from (1.10) when $1<p<\infty$, because $\dot{W}_{p}^{1}(\Omega)=\left(L_{p}(\Omega), \dot{W}_{p}^{2}(\Omega)\right)_{1 / 2, \infty}$ in this case. This is not true when $p=1, \infty$ (cf. [9]), which is the reason for our indirect proof of the special case $m=1, k=0$ of (3.9).

We now consider the homogeneous equation with nonsmooth initial data.

Theorem 3.5. Assume that $f(x, t) \equiv 0$. Let $u$ be the solution of (1.5) and $u_{h}$ the solution of (1.6) with $u_{0 h}=P_{h} u_{0}$. Then

$$
\begin{aligned}
\left\|u_{h}(t)-u(t)\right\|_{p} & \leq C h^{r} t^{-r / 2}\left\|u_{0}\right\|_{p}, & & t>0, u_{0} \in L_{p}(\Omega), \quad 1 \leq p \leq \infty, \\
\left\|u_{h}(t)-u(t)\right\|_{\infty} & \leq C h^{r} t^{-(r+1) / 2}\left\|u_{0}\right\|_{\mathscr{M}}, & & t>0, u_{0} \in \mathscr{M}(\Omega) .
\end{aligned}
$$

Proof. Since $u(t)=E(t) u_{0}$ and $u_{h}(t)=E_{h}(t) P_{h} u_{0}$, we must estimate the norm of the error operator $F_{h}(t)=E_{h}(t) P_{h}-E(t)$. We first consider (3.6) again and divide the interval of integration into $(0, t / 2)$ and $(t / 2, t)$. Integrating by parts in the first integral, noting that $P_{h} e(0)=0$, we get

$$
\theta(t)=-E_{h}(t / 2) P_{h} \rho(t / 2)-\int_{0}^{t / 2} E_{h}^{\prime}(t-s) P_{h} \rho(s) d s-\int_{t / 2}^{t} E_{h}(t-s) P_{h} \rho_{s}(s) d s .
$$

Using the stability estimates in (1.11) and the error estimates (3.5), we obtain

$$
\begin{aligned}
\|\theta(t)\|_{p} & \leq C\|\rho(t / 2)\|_{p}+C \int_{0}^{t / 2}(t-s)^{-1}\|\rho(s)\|_{p} d s+C \int_{t / 2}^{t}\left\|\rho_{s}(s)\right\|_{p} d s \\
& \leq C h^{k}\left(\|u(t / 2)\|_{k, p}+t^{-1} \int_{0}^{t / 2}\|u(s)\|_{k, p} d s+\int_{t / 2}^{t}\left\|u_{s}(s)\right\|_{k, p} d s\right),
\end{aligned}
$$

for $k=1, \ldots, r$. In particular, it follows that

$$
\left\|F_{h}(t) u_{0}\right\|_{p} \leq C h^{r} \sup _{0 \leq s \leq t}\left(\left\|E(s) u_{0}\right\|_{r, p}+s\left\|E^{\prime}(s) u_{0}\right\|_{r, p}\right) .
$$

Further, by (3.7) with $m=1$, we obtain a preliminary estimate of low order:

$$
\begin{aligned}
\left\|F_{h}(t) u_{0}\right\|_{p} & \leq C h t^{-1 / 2} \sup _{0<s<t}\left(s^{1 / 2}\left\|E(s) u_{0}\right\|_{1, p}+s^{3 / 2}\left\|E^{\prime}(s) u_{0}\right\|_{1, p}\right) \\
& \leq C h t^{-1 / 2}\left\|u_{0}\right\|_{p} .
\end{aligned}
$$

Writing

$$
F_{h}(t) u_{0}=F_{h}(t / 2) E(t / 2) u_{0}+E(t / 2) F_{h}(t / 2) u_{0}+F_{h}(t / 2)^{2} u_{0}
$$


we have, by (3.11), (3.7) with $m=r$,

$$
\begin{aligned}
\left\|F_{h}(t / 2) E(t / 2) u_{0}\right\|_{p} & \leq C h^{r} \sup _{t / 2 \leq s \leq t}\left(\left\|E(s) u_{0}\right\|_{r, p}+s\left\|E^{\prime}(s) u_{0}\right\|_{r, p}\right) \\
& \leq C h^{r} t^{-r / 2}\left\|u_{0}\right\|_{p},
\end{aligned}
$$

and in view of (3.8), using $t / 4$ as an intermediate level,

$$
\left\|F_{h}(t / 2) E(t / 2) u_{0}\right\|_{\infty} \leq C h^{r} t^{-(r+1) / 2}\left\|u_{0}\right\|_{\mathscr{M}} .
$$

We further note that $E(t / 2) F_{h}(t / 2)$ is the adjoint of the operator $F_{h}(t / 2)^{*} E(t / 2)^{*}$, where $E(t)^{*}=e^{-t A^{*}}$ and similarly for $F_{h}(t)^{*}$. Since the operator $F_{h}(t / 2)^{*} E(t / 2)^{*}$ is bounded in $L_{p}(\Omega)$ for $1 \leq p \leq \infty$ as in (3.14), its adjoint is similarly bounded in $L_{p^{\prime}}(\Omega), p^{\prime}$ being the conjugate exponent to $p$. Hence,

$$
\left\|E(t / 2) F_{h}(t / 2) u_{0}\right\|_{p} \leq C h^{r} t^{-r / 2}\left\|u_{0}\right\|_{p} .
$$

For the third term in (3.13) we have by (3.12)

$$
\left\|F_{h}(t / 2)^{2} u_{0}\right\|_{p} \leq C h t^{-1 / 2}\left\|F_{h}(t / 2) u_{0}\right\|_{p},
$$

and we may conclude that altogether

$$
\left\|F_{h}(t) u_{0}\right\|_{p} \leq C h^{r} t^{-r / 2}\left\|u_{0}\right\|_{p}+C h t^{-1 / 2}\left\|F_{h}(t / 2) u_{0}\right\|_{p},
$$

or, after $r$ iterations,

$$
\left\|F_{h}(t) u_{0}\right\|_{p} \leq C h^{r} t^{-r / 2}\left(\left\|u_{0}\right\|_{p}+\left\|F_{h}\left(t / 2^{r}\right) u_{0}\right\|_{p}\right) .
$$

Since, by stability $\left\|F_{h}\left(t / 2^{r}\right) u_{0}\right\|_{p} \leq C\left\|u_{0}\right\|_{p}$, we infer that

$$
\left\|F_{h}(t) u_{0}\right\|_{p} \leq C h^{r} t^{-r / 2}\left\|u_{0}\right\|_{p},
$$

which proves the first inequality of the theorem.

Finally, we consider the case when $u_{0} \in \mathscr{M}(\Omega)$. For the second term on the right in (3.13) we then have by (3.8)

$$
\left\|E(t / 2) F_{h}(t / 2) u_{0}\right\|_{\infty} \leq C t^{-1 / 2}\left\|E(t / 4) F_{h}(t / 2) u_{0}\right\|_{\mathscr{K}} .
$$

Here, $E(t / 4) F_{h}(t / 2)$ is the adjoint of the operator $F_{h}(t / 2)^{*} E(t / 4)^{*}$. Since the operator $F_{h}(t / 2)^{*} E(t / 4)^{*}: L_{\infty}(\Omega) \rightarrow C(\bar{\Omega})$ is bounded as in (3.14), its adjoint is similarly bounded in $\mathscr{M}(\Omega)$. In fact,

$$
\left(E(t / 4) F_{h}(t / 2) u_{0}, \phi\right)=\left\langle u_{0}, F_{h}^{*}(t / 2) E^{*}(t / 4) \phi\right\rangle \leq\left\|u_{0}\right\|_{\mathscr{M}}\left\|F_{h}^{*}(t / 2) E^{*}(t / 4) \phi\right\|_{\infty},
$$

for all $u_{0} \in \mathscr{M}(\Omega), \phi \in C(\bar{\Omega})$. Hence,

$$
\left\|E(t / 2) F_{h}(t / 2) u_{0}\right\|_{\infty} \leq C h^{r} t^{-(r+1) / 2}\left\|u_{0}\right\|_{\mathscr{M}} .
$$

The proof can now be completed in the same way as above, using the inequality

$$
\left\|F_{h}\left(t / 2^{r}\right) u_{0}\right\|_{\infty} \leq C t^{-1 / 2}\left\|u_{0}\right\|_{\mathscr{M}},
$$

which follows from (3.8).

We conclude by proving the error bound (1.22) for a completely discrete approximation of the solution of the homogeneous problem, which we announced in the Introduction. 
Theorem 3.6. Let $u$ be the solution of (1.5) with $f(x, t) \equiv 0$ and let $U_{n}=$ $r\left(-k A_{h}\right)^{n} u_{0 h}$. Assume that the rational function $r$ is accurate of order $q$ (1.15) and A-acceptable (1.16). Then, for $1 \leq p \leq \infty$, we have

$$
\left\|U_{n}-u\left(t_{n}\right)\right\|_{p} \leq C\left(\left\|u_{0 h}-u_{0}\right\|_{p}+h^{r}\left\|u_{0}\right\|_{r, p}+k^{q}\left\|u_{0}\right\|_{2 q, p}\right), \quad t_{n}=n k \geq 0,
$$

if $u_{0} \in \dot{W}_{p}^{m}(\Omega)$ with $m=\max (r, 2 q)$.

Proof. By stability (cf. (1.17)) there is no loss of generality in assuming that $u_{0 h}=P_{h} u_{0}$. Using (3.11) and (3.9), we obtain

$$
\left\|E_{h}\left(t_{n}\right) P_{h} u_{0}-E\left(t_{n}\right) u_{0}\right\|_{p} \leq C h^{r}\left\|u_{0}\right\|_{r, p},
$$

and it remains to estimate

$$
G_{n} P_{h} u_{0} \equiv\left(r\left(-k A_{h}\right)^{n}-E_{h}\left(t_{n}\right)\right) P_{h} u_{0} .
$$

Following [1], we use the identity

$$
\begin{aligned}
G_{n} P_{h} u_{0}= & \sum_{j=0}^{q-1} G_{n} A_{h}^{-j}\left(P_{h} A^{-1}-A_{h}^{-1} P_{h}\right) A^{j+1} \tilde{u}_{0 k} \\
& +G_{n} A_{h}^{-q} P_{h} A^{q} \tilde{u}_{0 k}+G_{n} P_{h}\left(u_{0}-\tilde{u}_{0 k}\right),
\end{aligned}
$$

where $\tilde{u}_{0 k}$ is to be chosen so that

$$
\begin{aligned}
\left\|u_{0}-\tilde{u}_{0 k}\right\|_{p} & \leq C k^{q}\left\|u_{0}\right\|_{2 q, p}, \\
\left\|A^{q} \tilde{u}_{0 k}\right\|_{p} & \leq C\left\|u_{0}\right\|_{2 q, p}, \\
\left\|A^{j} \tilde{u}_{0 k}\right\|_{r, p} & \leq C k^{-j}\left\|u_{0}\right\|_{r, p}, \quad 0 \leq j \leq q-1 .
\end{aligned}
$$

In [1], $\tilde{u}_{0 k}$ is defined by truncating the Fourier series of $u_{0}$, but this is not suitable here and we choose instead

$$
\tilde{u}_{0 k}=f(k A) E(k A) u_{0}, \quad \text { where } f(\lambda) \equiv \sum_{n=0}^{q}(-\lambda)^{n} / n !=e^{-\lambda}+O\left(\lambda^{q+1}\right), \lambda \rightarrow 0,
$$

so that $1-f(\lambda) e^{\lambda}=O\left(\lambda^{q+1}\right)$, as $\lambda \rightarrow 0$. Hence, using the same contour $\Gamma$ as in (1.8) and the resolvent estimate of Theorem 1.1, we have

$$
\begin{aligned}
\left\|u_{0}-\tilde{u}_{0 k}\right\|_{p} & =\left\|A^{-q}(I-f(k A) E(k A)) A^{q} u_{0}\right\|_{p} \\
& =\left\|\frac{1}{2 \pi i} \int_{\Gamma} \lambda^{-q}\left(1-f(k \lambda) e^{k \lambda}\right) R(\lambda,-A) d \lambda A^{q} u_{0}\right\|_{p} \\
& =k^{q-1}\left\|\frac{1}{2 \pi i} \int_{\Gamma} \lambda^{-q}\left(1-f(\lambda) e^{\lambda}\right) R\left(k^{-1} \lambda,-A\right) d \lambda A^{q} u_{0}\right\|_{p} \\
& \leq C k^{q} \int_{0}^{\infty} \rho^{-q-1}\left|1-f\left(\rho e^{i \varphi}\right) \exp \left(\rho e^{i \varphi}\right)\right| d \rho\left\|A^{q} u_{0}\right\|_{p} \\
& \leq C k^{q}\left\|u_{0}\right\|_{2 q, p},
\end{aligned}
$$

where we also used the fact that $A$ is a second-order differential operator. This is (3.17). Moreover, by the analyticity of $E(t)$, see (1.10), we have

$$
\begin{aligned}
\left\|A^{q} \tilde{u}_{0 k}\right\|_{p} & =\left\|A^{q} f(k A) E(k A) u_{0}\right\|_{p} \leq \sum_{n=0}^{q} \frac{1}{n !}\left\|(k A)^{n} E(k A) A^{q} u_{0}\right\|_{p} \\
& \leq C\left\|A^{q} u_{0}\right\|_{p} \leq C\left\|u_{0}\right\|_{2 q, p},
\end{aligned}
$$


which is (3.18). Using also the smoothing property (3.9), we obtain

$$
\begin{aligned}
\left\|A^{j} \tilde{u}_{0 k}\right\|_{r, p} & =k^{-j}\left\|(k A)^{j} f(k A) E(k A) u_{0}\right\|_{r, p} \\
& \leq k^{-j} \sum_{n=0}^{q} \frac{1}{n !}\left\|(k A)^{n+j} E(k A) u_{0}\right\|_{r, p} \leq C k^{-j}\left\|u_{0}\right\|_{r, p},
\end{aligned}
$$

which is (3.19).

Returning now to the identity (3.16), we first note that (1.17), (1.20), and (1.11) imply

$$
\left\|G_{n} A_{h}^{-j} v_{h}\right\|_{p} \leq C k^{j}\left\|v_{h}\right\|_{p}, \quad 0 \leq j \leq q, v_{h} \in S_{h} .
$$

Moreover, since $A_{h}^{-1} P_{h}=R_{h} A^{-1}$, Lemma 3.1 implies

$$
\left\|\left(P_{h} A^{-1}-A_{h}^{-1} P_{h}\right) v\right\|_{p}=\left\|P_{h}\left(I-R_{h}\right) A^{-1} v\right\|_{p} \leq C h^{r}\left\|A^{-1} v\right\|_{r, p} .
$$

Hence, using also (3.19), we find

$$
\begin{aligned}
\left\|G_{n} A_{h}^{-j}\left(P_{h} A^{-1}-A_{h}^{-1} P_{h}\right) A^{j+1} \tilde{u}_{0 k}\right\|_{p} & \leq C k^{j}\left\|\left(P_{h} A^{-1}-A_{h}^{-1} P_{h}\right) A^{j+1} \tilde{u}_{0 k}\right\|_{p} \\
& \leq C k^{j} h^{r}\left\|A^{j} \tilde{u}_{0 k}\right\|_{r, p} \leq C h^{r}\left\|u_{0}\right\|_{r, p},
\end{aligned}
$$

for $0 \leq j \leq q-1$. Similarly, using (3.20) with $j=q$ and (3.18), we have

$$
\left\|G_{n} A_{h}^{-q} P_{h} A^{q} \tilde{u}_{0 k}\right\|_{p} \leq C k^{q}\left\|A^{q} \tilde{u}_{0 k}\right\|_{p} \leq C k^{q}\left\|u_{0}\right\|_{2 q, p} .
$$

Finally, by (3.20) with $j=0$ and (3.17), we conclude that

$$
\left\|G_{n} P_{h}\left(u_{0}-\tilde{u}_{0 k}\right)\right\|_{p} \leq C\left\|u_{0}-\tilde{u}_{0 k}\right\|_{p} \leq C k^{q}\left\|u_{0}\right\|_{2 q, p},
$$

and we have estimated all the terms in (3.16) in the desired way.

\section{BIBLIOGRAPHY}

1. G. A. Baker, J. H. Bramble, and V. Thomée, Single step Galerkin approximations for parabolic problems, Math. Comp. 31 (1977), 818-847.

2. P. Brenner, M. Crouzeix, and V. Thomée, Single step methods for inhomogeneous linear differential equations in Banach space, RAIRO Anal. Numér. 16 (1982), 5-26.

3. M. Crouzeix, S. Larsson, S. Piskarev, and V. Thomée, The stability of rational approximations of analytic semigroups, BIT 33 (1993), 74-84.

4. M. Crouzeix and V. Thomée, The stability in $L_{p}$ and $W_{p}^{1}$ of the $L_{2}$-projection onto finite element function spaces, Math. Comp. 48 (1987), 521-532.

5. G. Da Prato and P. Grisvard, Sommes d'opérateurs linéaires et équations différentielles opérationnelles, J. Math. Pures Appl. 54 (1975), 305-387.

6. G. Da Prato and E. Sinestrari, Differential operators with non dense domain, Ann. Scuola Norm. Sup. Pisa Cl. Sci. (4) 14 (1987), 285-344.

7. J. Douglas, Jr., T. Dupont, and L. Wahlbin, Optimal $L_{\infty}$ error estimates for Galerkin approximations to solutions of two-point boundary value problems, Math. Comp. 29 (1975), 475-483.

8. S. Larsson, V. Thomée, and L. B. Wahlbin, Finite-element methods for a strongly damped wave equation, IMA J. Numer. Anal. 11 (1991), 115-142.

9. B. S. Mitjagin and E. M. Semenov, Absence of interpolation of linear operators in spaces of smooth functions, Izv. Akad. Nauk SSSR Ser. Mat. 41 (1977), 1289-1328; English transl. in Math. USSR Izv. 11 (1977), 1229-1266.

10. C. Palencia, A stability result for sectorial operators in Banach spaces, SIAM J. Numer. Anal. 30 (1993), 1373-1384. 
11. _ On the stability of variable stepsize rational approximations of holomorphic semigroups, Math. Comp. 62 (1994), 93-103.

12. A. Pazy, Semigroups of linear operators and applications to partial differential equations, Springer-Verlag, 1983.

13. E. Sinestrari, On the abstract Cauchy problem of parabolic type in spaces of continuous functions, J. Math. Anal. Appl. 107 (1985), 16-66.

14. V. Thomée and L. B. Wahlbin, Maximum-norm stability and error estimates in Galerkin methods for parabolic equations in one space variable, Numer. Math. 41 (1983), 345-371.

15. L. B. Wahlbin, A quasioptimal estimate in piecewise polynomial Galerkin approximation of parabolic problems, Numerical methods, Proceedings, Dundee 1981 (G. A. Watson, ed.), Lecture Notes in Math., vol. 912, Springer-Verlag, 1981, pp. 230-245.

(M. Crouzeix) IRMAR, Université de Rennes I, Campus de Beaulieu, F-35042 Rennes Cedex, France

E-mail address: crouzeix@irisa.fr

(S. Larsson and V. Thomée) Department of Mathematics, Chalmers University of TechNOLOGY AND THE UNIVERSITY OF GöteboRg, S-412 96 GöteboRG, SwedeN

$E$-mail address, S. Larsson: stig@math . chalmers. se

E-mail address, V. Thomée: thomee@math.chalmers.se 